Original Research Paper

\title{
Reinforcing Effect of Strain Gauges on 3D Printed Polymers: An Experimental Investigation
}

\author{
Roberto Torre and Salvatore Brischetto \\ Department of Mechanical and Aerospace Engineering, Politecnico di Torino, Torino, Italy
}

\author{
Article history \\ Received: 17-11-2020 \\ Revised: 05-01-2021 \\ Accepted: 08-01-2021 \\ Corresponding Author: \\ Roberto Torre \\ Department of Mechanical and \\ Aerospace Engineering, \\ Politecnico di Torino, Torino, \\ Italy \\ Email: roberto.torre@polito.it
}

\begin{abstract}
Strain gauges are ordinary transducers for strain measuring; their operation relies on the electrical resistance, which varies as the underlying substrate is subjected to mechanical deformation. The mechanical strain can be obtained by converting the electrical signal through a gauge factor, provided by the manufacturer. It was demonstrated that its values are not unique; it may be influenced by the geometrical characteristics of both the specimen and the strain gauge and by their respective moduli of elasticity. This can be extremely dangerous when low modulus materials are studied. This study confirms that even with commercial strain gauges specifically designed for low modulus materials the effect might be present. An experimental method for its evaluation is discussed; tensile specimens are used as a test bench and their modulus is determined using both strain gauges and a noncontact method (Digital Image Correlation). The results show that a local reinforcing effect is present and a higher tensile modulus is obtained when contact transducers are bonded to polymeric specimens. The amplitude of this effect is predicted with established methods available in the literature and through a simple 2D Finite Element (FE) model. All these models require the elastic modulus of the strain gauge to be considered; a digital procedure to estimate it for any wired transducer is therefore proposed. The predicted results were found to be consistent with those experimentally measured; this validated the method, thus advising on how to evaluate the phenomenon also when this information is not available.
\end{abstract}

Keywords: Strain Gauge, Polymer, Material Testing, Material Characterization, Digital Image Correlation

\section{Introduction}

Stress analysis is mostly performed by measuring the strain field over the component under investigation. A strain field evaluation is also necessary to perform material characterization. In those circumstances, it is vital that precise measuring is performed. Consequently, lots of authors worked on new measuring techniques and aimed to improve the existing ones. Several instruments and several techniques exist in this respect; some of them involve contact between the surface and the instrument, others keep at a distance (Arena and Viscardi, 2020). Among the most commonly used tools, strain gauges are a common choice (Kobayashi, 1987). However, it has been demonstrated that their installation may affect the strain field, leading to incorrect results if this aspect is not taken into account (Zike and Mikkelsen, 2014). Clip-on extensometers can be used alternatively, however, the indentation of the instrument might induce stress concentrations. In the frame of contact-devices, some authors proposed alternative instruments. Embedded Fiber Bragg Grating (FBG) were considered in (Pereira et al., 2016) for polymer characterization; in this study, the author used those sensors in tensile tests of dog-bone specimens and proposed a calibration protocol using extensometers. FBG own the advantage to not influence the material resistance and characteristics, but their installation can not be done on existing components and has to be considered before manufacturing (Montazerian et al., 2020). Non-contact-systems such as laser extensometers, video extensometers (Tian et al., 2018) and Digital 
Image Correlation system avoid any influence on the specimen (Jerabek et al., 2010). Those instruments have been successfully used in several applications (Muniandy et al., 2019; Liu et al., 2019), however, they are not free of downsides as the surface to be monitored needs to be in sight, stationary with respect to the measuring instrument. More important, sufficient clearance is needed between the measuring instrument and the specimen.

Strain gauges continue to be used in several applications; those transducers are based on the change in resistance of electrical wires, subjected to a load (Hoffmann, 1989). To ensure this effect is measurable and to keep the transducer's dimensions small, the electrical wire takes a specific pattern which emphasizes its sensitivity in a specific direction. Metal-foil strain gauges are the most common; they are made of a metallic foil encapsulated into a backing material, subsequently glued onto the surface to be monitored (Watson, 2008). The manufacturers provide the user a so-called gage factor, $\mathrm{Sg}$, which represents the calibration constant obtained over a huge sample of identical transducers, which allows the percentage change in resistance to be converted into a strain. Several authors investigated the effect the strain gauge installation produces on the component. When materials with a high elastic modulus are studied and when the dimensions of the component are relevant with respect to those of the strain gauge, the effect is negligible (Kobayashi, 1987). However, when low modulus materials (Dove, 1955; Clark, 1956) or thin samples (Campbell and Medbery, 1947) are studied it has been demonstrated that the strain field around the installation area may be affected. For benchmark, (Kobayashi, 1987) discusses that a classical strain gauge manifests an effective modulus of elasticity in the range 7-20 GPa. Little et al. (1990) discussed that the reinforcement due to the bonded strain gauge is a function of several parameters, which range from the self-heating effect to the gauge type, also including the plastic material properties and the lead wires.

It is well established in the literature that two effects might be detected while studying a strain gauge installation. The local reinforcing effect manifests in a modification of the strain field near the region the strain gauge has been applied to; it occurs when the cross-section of the specimen is large and its constituent has a low modulus of elasticity. The global reinforcing effect occurs when the strain field is modified in the whole cross-section of the specimen; it is common in small cross-section items (Xue et al., 2018). Those effects can be also found in different transducers, for different applications, which need to be mounted on the element (Borri-Brunetto et al., 2016) to be studied.

Stehlin (1972) among the first studied the phenomenon, discussing the mutual interaction of the substrate with the glued strain gauge. The strain distribution in the installation area was studied for several materials; a model for strain distortion was proposed and a photo-elastic experiment was carried out to see strain-perturbations. Beatty and Chewning (1979) conducted a numerical analysis, to determine the influence of geometrical and dimensional characteristics of strain gauge. The authors determined that an increase in the length and a decrease in the thickness of the gage has a positive effect in reducing the reinforcing effect; also decreasing the thickness of the adhesive layer plays a reliving role, if little. An empirical formula was proposed, which relates the strain measured by the transducer $\varepsilon^{\prime}$ and the actual strain in the specimen $\varepsilon$ :

$\frac{\varepsilon^{\prime}}{\varepsilon}=1-r \alpha-s \beta$

$\alpha$ and $\beta$ describe the local reinforcing effect in terms of geometrical and mechanical characteristics of both the specimen and the strain gauge:

$\alpha=\frac{4 t_{s g}}{L_{s g}} \frac{E^{s g}}{E^{s}}$

$\beta=\frac{t_{a}}{L_{s g}} \frac{E^{s}}{G^{a}} \alpha$

where, $t_{s g}$ and $L_{s g}$ are the thickness of the strain gauge and its total length, $E^{s g}$ and $E^{s}$ are the elastic moduli of the strain gauge and of the specimen, $t_{a}$ and $G^{a}$ are the thickness and the shear modulus of the adhesive layer. $r$ and $s$ of Eq. 1 depend on the ratio between the length of the active part of the strain gauge $L_{g}$ (gauge length) and the overall length $L_{s g}$.

A theoretical model has been proposed by (Ajovalasit and Zuccarello, 2005); the strain gauge was considered to be installed on a plate with semi-infinite dimensions. A closed-form solution was found disregarding the effect of the adhesive and assuming an exponential distribution for the shear stress at the interface. The outcome of the model is analogous to the previous one, as it is described by the following relation: 


$$
\frac{\varepsilon^{\prime}}{\varepsilon}=1-\frac{t_{s g}}{L_{s g}} \frac{E^{s g}}{E^{s}} \phi\left(\frac{L_{s g}}{t_{s g}}, \frac{L_{g}}{t_{s g}}\right)
$$

The same authors showed in (Ajovalasit et al., 2007) that not only the local reinforcement effect grabbed the attention of researchers; in this study, it was discussed that the overall effects produced by the strain gauge installation are better described in terms of the apparent modulus of elasticity of the substrate, obtained considering the strain measurements made by the transducer. In the experimental analysis, it was also determined that the stiffness of the gauge is not significantly affected by the type of adhesive employed. Back in time, (Swan, 1973) already discussed a way to predict the global reinforcement effect. Perry (1989) analyzed the combination of the two effects, discussing that in intermediate configurations both might be present. A model aimed to compensate for the reinforcement on low modulus materials was proposed. In general, all the authors that studied the phenomenon warned the operators that significant errors might arise if the reinforcing effects are not properly compensated. In (Zike and Mikkelsen, 2014) the correction coefficients for a wide range of elastic moduli of the substrate and a wide range of relations between the specimen/strain gauge dimensions was determined. Significant values were found even for sufficiently stiff specimens. The simulations were validated through experimental tests on polymer matrix fiber reinforced polymers with different elastic moduli. A detailed Finite Element (FE) 3D model was used for correction coefficients prediction; the study focused on a specific strain gauge model. Nevertheless, it should be considered that in some specific situations the ability to simulateg such effect might not be sufficient to correct it (Shen et al., 2013).

The analytical models discussed in (Ajovalasit and Zuccarello, 2005; Beatty and Chewning, 1979) give a practical formula to evaluate the reinforcing effect. However, its determination is subject to the quantification of strain gauge elastic modulus, which is a detail difficult to achieve practically. At the same time, the numerical model discussed in (Zike and Mikkelsen, 2014) needs the same value and it is difficult to generalize to any strain gauge/specimen combination. This study arose in the frame of wider research on the mechanical behavior of polymeric elements produced via Fused Deposition Modelling/Fused Filament Fabrication (FDM/FFF) currently conducted by the authors. (Brischetto et al., 2017; Brischetto and Torre, 2020). A further study of the reinforcing effect is here discussed, proposing an experimental and a numerical method of evaluating and predicting it. The quantification of the reinforcing effect is performed using the Digital Image Correlation (DIC) technique as a benchmark, given its non-contact capabilities of strain measuring. In parallel, a 2D Finite Element (FE) model is proposed for reinforcement prediction. As the phenomenon relies on the mechanical properties of the strain gauge in use, which are hardly known to final user, a digital procedure is proposed for their evaluation. All the study is based on commercially strain gauges, manufactured by Tokyo Measuring Instruments Laboratory Co., Ltd., specifically designed for low modulus materials.

\section{Experimental Set-Up}

Section discussed the reinforcement effect, whether local or global, by referring to established previous works in the literature. This section is devoted to the experimental campaign conducted in order to analyze and quantify the phenomenon under specific circumstances. The idea is that each strain gauge manifests an apparent modulus of elasticity, which depends on the characteristics of its constituents and on its mesostructure. When this apparent modulus of elasticity is higher than the one of the specimen the strain measurements of the transducer may be influenced by this scenario. Both the local effect and the global effect are quantified.

3D printed Poly Lactic Acid (PLA) was considered as testing material; a black filament manufactured by Shenzhen Eryone Technology Co., Ltd. was used. Table 1 reports the data-sheet containing the characteristics of the raw material (Shenzhen Eryone Technology, 2019) for reference; they cannot be considered representative of the processed PLA as FDM/FFF techniques are known to affect them through their processing parameters (Moradi et al., 2020). To isolate the stiffening effect, a tensile test was selected as test bench as it is designed to produce a uniform stress distribution inside the coupon. In this way, it was possible to evaluate the phenomenon in terms of apparent modulus of elasticity. Regardless of the test method, the tensile modulus of elasticity is determined from the region of the stress-strain curve in which a linear relation holds between the two variables. As discussed in (Ajovalasit et al., 2007), this quantity is a better benchmark than the simple strain and allows to get a better understanding of the phenomenon. Torre et al. (2018), the authors outlined a parallel between 3D printed components with a linear infill and long fiber composites. Constant rectangular cross-section specimens were therefore used, with bonded tabs on both specimen's ends, following the standard test method for tensile properties determination ASTM D3039 (ASTM, 2017). To evaluate the reinforcing effect, the strain gauge measurements needed to be compared to those obtained via a different 
technique. A non-contact strain measuring system, the Digital Image Correlation (DIC) technique was used as a reference measuring "device" for strains evaluation. A total number of 8 specimens were printed, divided into 2 runs of 4 specimens each; the main printing parameters are summarized in Table 2. The experimental set-up was differently planned among the two runs in order to focus on different targets. The specimens of both the run were tested in the same way, however:

- $\operatorname{Run}(\mathrm{A})$ specimens were monitored via DIC only

- Run(B) specimens were monitored both via SG and DIC

Run(A) specimens were not influenced by any strain measuring device as the longitudinal strains were measured without contact, with the DIC system; those specimens will be referred to as Free $(F)$ specimens. Run(B) specimens deformed under tensile load together with their bonded strain gauges; hereinafter reference will be made to Instrumented (I) specimen when discussing about them. After each strain measurement had been synced with the corresponding stress measurement, a Matlab tool was developed to determine the slope of the linear part of the stress-strain relation. Run(A) specimens led to the determination of the actual modulus of elasticity of 3D printed PLA. Run(B) specimens led to the evaluation of two apparent modulus of elasticity as two sets of strains could be used for the same specimens, DIC-measured and SG-measured. The comparison of DIC-measured Run(B) specimens Vs. DICmeasured Run(A) specimens allowed the global reinforcing effect to be evaluated; the comparison of SG-measured Run(B) specimens Vs. DIC-measured Run(B) specimens allowed the local reinforcing effect to be evaluated.

\section{Tensile Tests}

This experimental investigation was conducted in the frame of wider research focused on the mechanical characterization of 3D printed PLA. For consistency, the geometrical features of the specimen and the mesostructure were not altered. As anticipated, the tensile tests were performed on rectangular cross-section specimens. Tabs were found to be necessary to make a uniform stress state develop inside the coupons and to limit stress concentrations; as a consequence, a set of four tabs was printed and bonded to each specimen. Bicomponent epoxy glue was used to bond specimens and tabs. The geometrical and dimensional features of a specimen with bonded tabs are reported in Fig. 1, where a 2D sketch is presented. The tensile tests were conducted with an MTS Qtest universal testing machine, equipped with a $10 \mathrm{kN}$ load cell. Each specimen was positioned in the grip of the lower (fixed) grip; the upper (movable) grip was then lowered to accommodate the specimen and then fastened. The test was conducted in displacement control, with the machine control system adapting the applied load in such a way to ensure that the upper grip moved with a speed of $5 \mathrm{~mm} / \mathrm{min}$ until each specimen failed. This testing speed was chosen as suggested for tensile properties measurement in (ASTM, 2017). During each test, the machine recorded the applied load and the imposed displacement. This allowed to draw the Load-Displacement curves shown in Figs. 2 and 3 for Run(A) and Figs. 4 and 5 for Run(B). Those curves are not strictly representative of the mechanical behavior of 3D printed PLA as:

- The load depends on the actual cross-section dimensions of the specimen

- The displacements are not homogeneously distributed across the specimens as they develop across the narrow section, but also across the sections under the beveled parts of the tabs. Furthermore, it is possible that the free length of each specimen differed from the others due to its manual placement in the testing apparatus

Despite what discussed, Figs. 2 and 5 allow to make some preliminary considerations. All the tested specimens manifested an initial linear trend of the load Vs. the displacement. A slight deviation from the linearity occurred shortly before the peak load was reached; a great scatter was found with respect to its value, which appeared to be very sensitive to the internal defects distribution. As appears from Fig. 5b, specimen I4 slipped during the test at a very low load. For this reason and as discussed below, it was excluded from the following analysis. As shown in Fig. 6 and 7 all the specimens broke inside the gage area; all of them broke abruptly, with a break line following mostly the filament distribution direction. As per (ASTM, 2017), the failure can be coded as Angled-Gage-Middle (AGM).

Table 1: Eryone black PLA data-sheet (Shenzhen Eryone Technology, 2019)

\begin{tabular}{lll}
\hline Density & $1.24 \mathrm{~g} / \mathrm{cm}^{3}$ & ASTM D792 \\
Tensile strength & $65 \mathrm{MPa}$ & ASTM D638 \\
Tensile yield & $60 \mathrm{MPa}$ & ASTM D638 \\
Young Modulus & $3950 \mathrm{MPa}$ & ASTM D638 \\
\hline
\end{tabular}

Table 2: Printing parameters used in Run(A) and Run(B) specimens production

\begin{tabular}{lll}
\hline 1st layer height & 0.20 & $\mathrm{~mm}$ \\
Gen. layer height & 0.10 & $\mathrm{~mm}$ \\
Nozzle diameter & 0.40 & $\mathrm{~mm}$ \\
Extrusion width & 0.50 & $\mathrm{~mm}$ \\
Infill pattern & linear & - \\
Infill percentage & $100 \%$ & - \\
Perimeters $\mathrm{n}^{\circ}$ & - & - \\
Raster angle sequence & {$\left[45^{\circ}\right]$} & - \\
Extrusion temperature & 190 & ${ }^{\circ} \mathrm{C}$ \\
Bed temperature & Room temp. & - \\
\hline
\end{tabular}



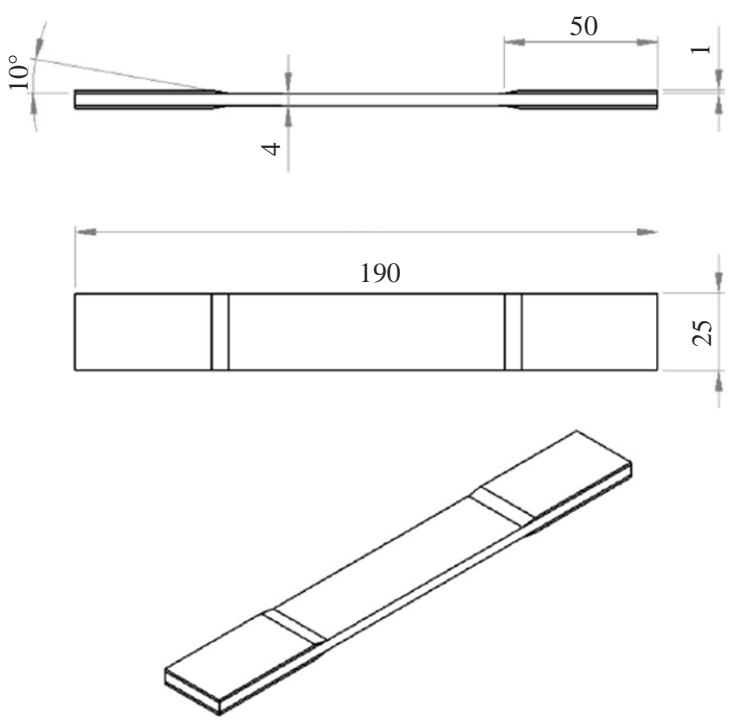

Fig. 1: Sketch of a tensile specimen with bonded tabs

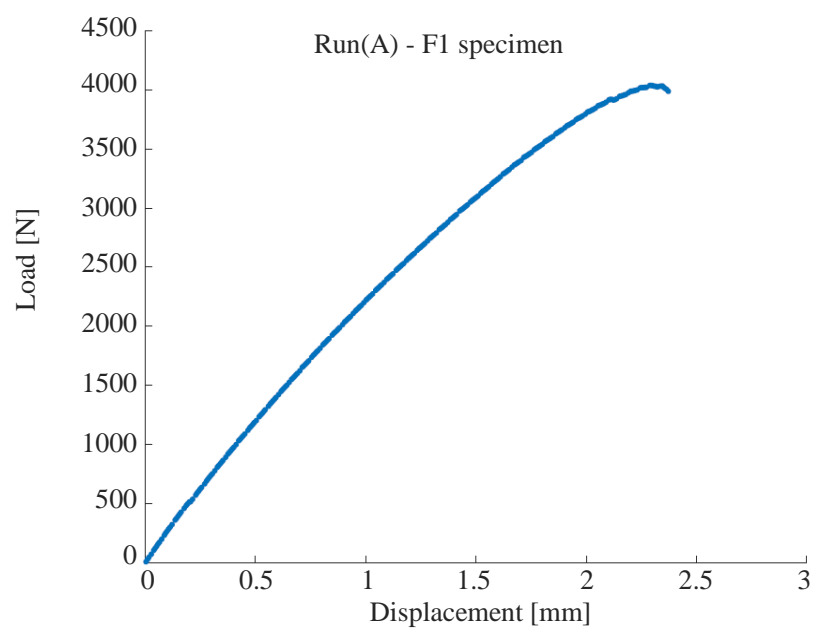

(a)

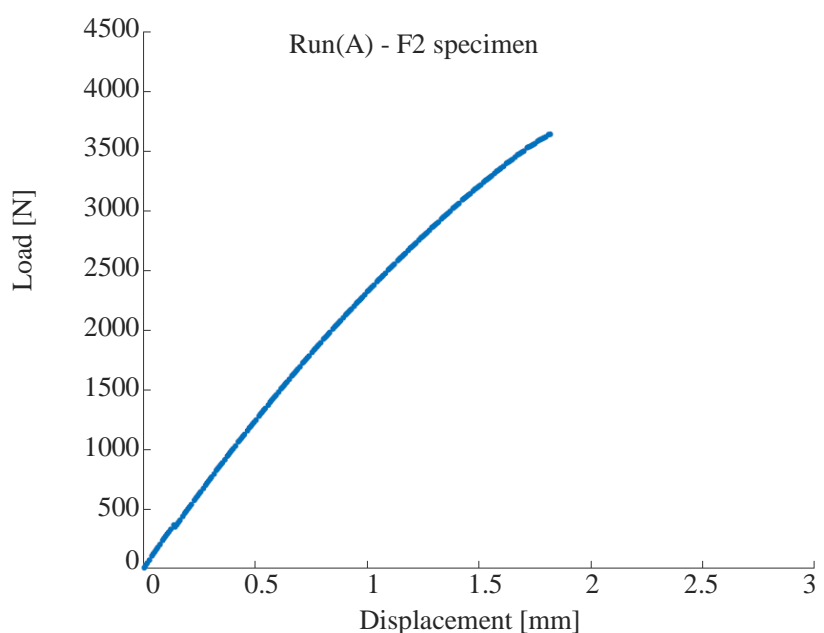

(b)

Fig. 2: Run(A): Free (F) specimens; tensile load Vs. longitudinal displacement curves; (a) Run(A) - F1; (b) Run(A) - F2 
Roberto Torre and Salvatore Brischetto / American Journal of Engineering and Applied Sciences 2021, 14 (1): 7.24 DOI: 10.3844/ajeassp.2021.7.24

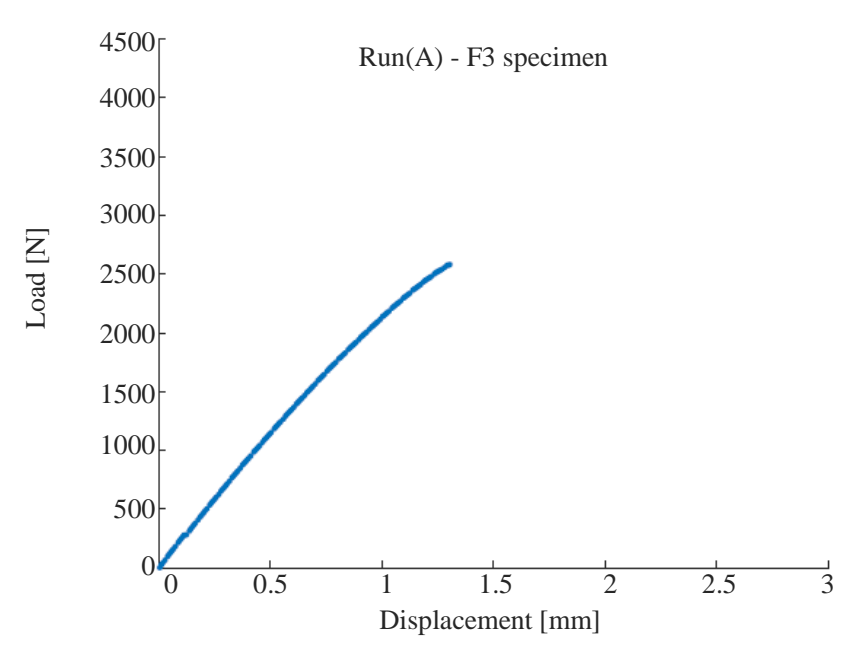

(a)

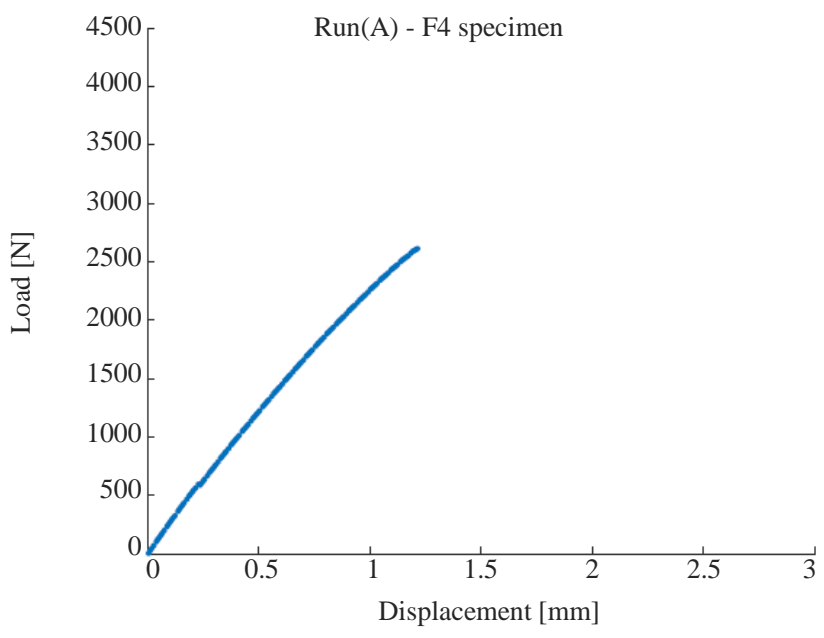

(b)

Fig. 3: Run(A): Free (F) specimens; tensile load Vs. longitudinal displacement curves; (a) Run(A) - F3; (b) Run(A) - F4

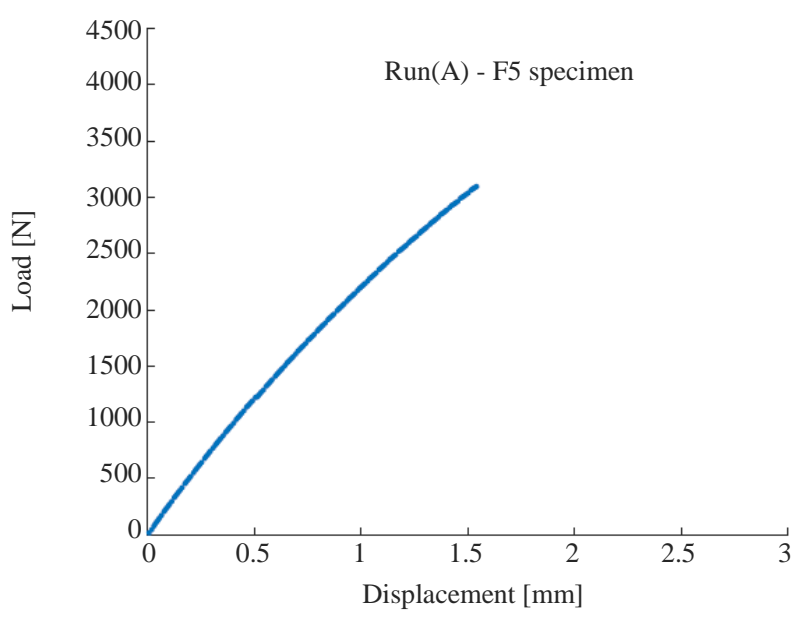

(a) 
Roberto Torre and Salvatore Brischetto / American Journal of Engineering and Applied Sciences 2021, 14 (1): 7.24 DOI: 10.3844/ajeassp.2021.7.24

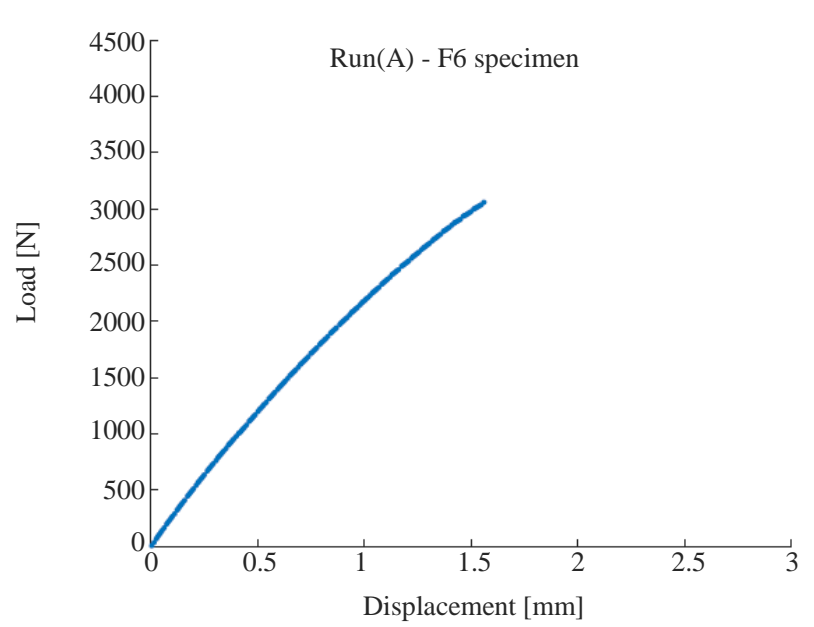

(b)

Fig. 4: Run(B): Instrumented (I) specimens; tensile load Vs. longitudinal displacement curves; (a) Run(B) - I1; (b) Run(B) - I2

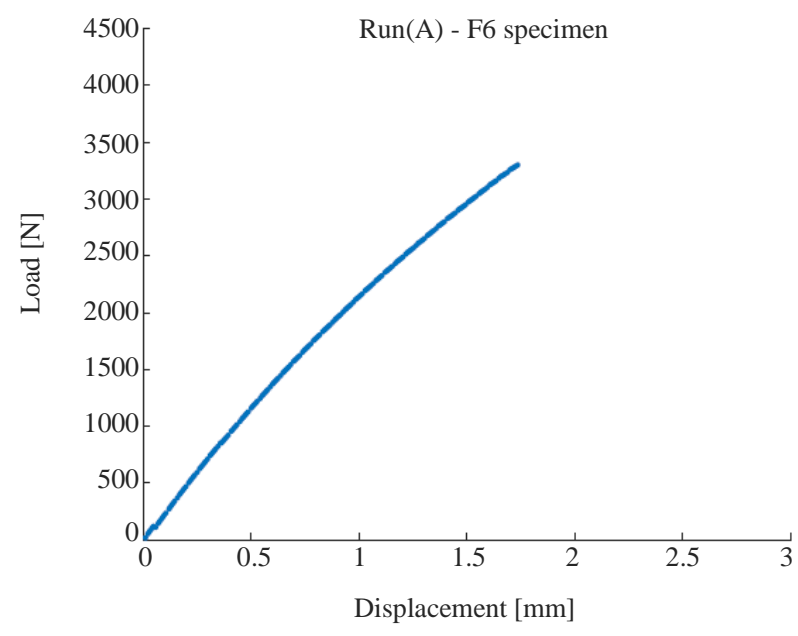

(a)

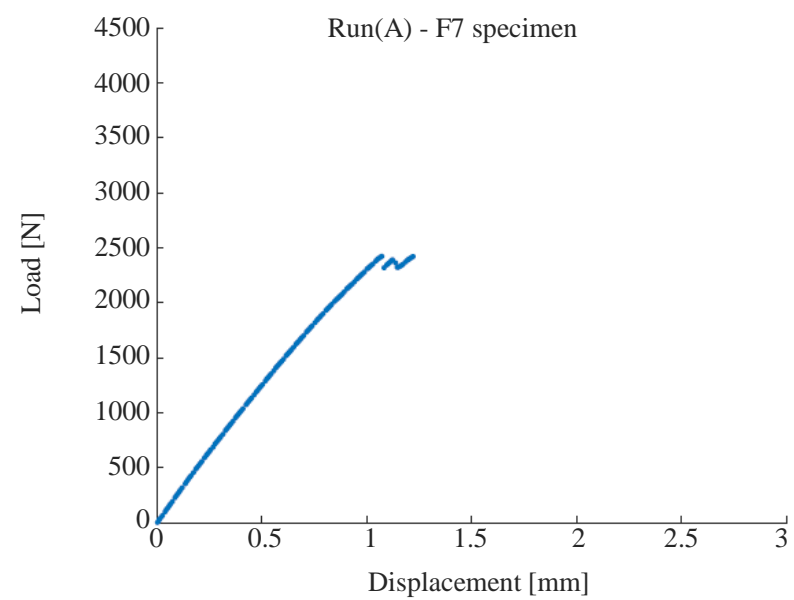

(b)

Fig. 5: Run(B): Instrumented (I) specimens; tensile load Vs. longitudinal displacement curves; (a) Run(B) - I3; (b) Run(B) - I4 


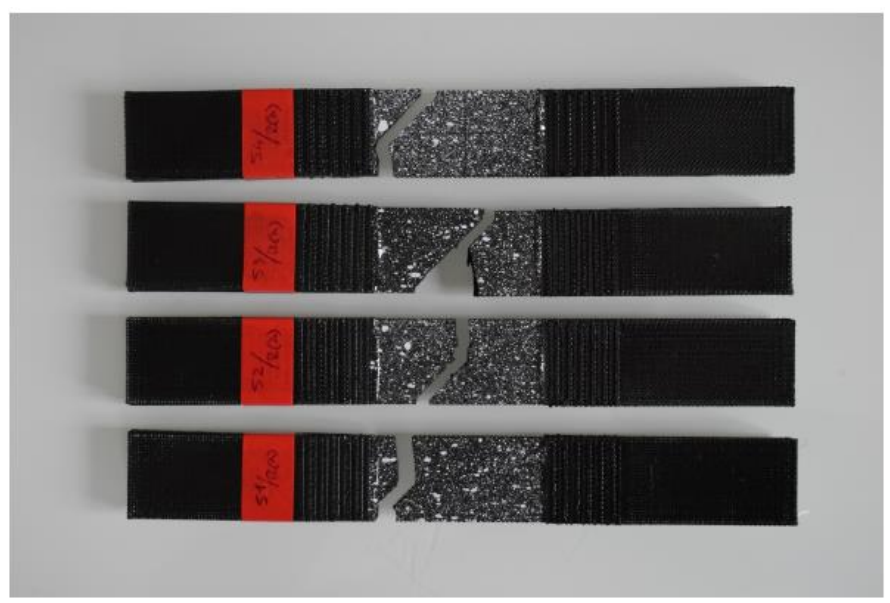

Fig. 6: Run(A) specimens after the tensile test ended

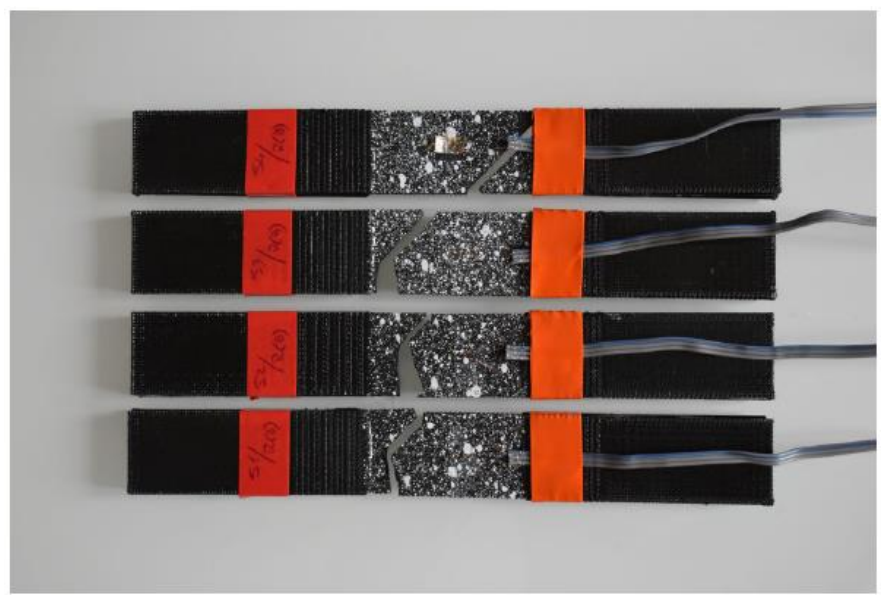

Fig. 7: Run(B) specimens after the tensile test ended

\section{Strain Gage Set-Up}

As anticipated, Run(B) specimens were monitored with SGs during the tests. Several configurations can be used to monitor the strains into a tensile specimen; by referring to longitudinal strains the following set-ups might be found in the literature:

- A single SG, symmetrically placed over the front surface of the specimen

- A pair of SGs, both placed over the front surface of the specimen at the same longitudinal coordinate and symmetrically with respect to the middle line

- A triple of SGs, of which the first two are positioned on the front surface as described in the previous setup and the latest on the back surface as described in the first set-up

The last two set-ups are meant to offset bending which may occur in the width plane (second set-up) or in the thickness plane and in the width plane (third set-up) due to an overall misalignment of the specimen and of the grips. As will be discussed in the next subsection, the DIC was carried out with a single camera focused on the front surface of the specimen; as a consequence, a SG positioned on the back surface would not have the basis of comparison. At the same time, an averaged front-back strain would have been inconsistent with the front strain calculated by DIC. Consequently, the third configuration was set aside. The second one also was shelved, in order to isolate the effect of a single SG, avoiding any kind of mutual influence or the accumulation of several sources of reinforcement. As suggested in ASTM D3039 (ASTM, 2017), the proper system alignment was evaluated, in order to limit data scatter. The percentage bending was evaluated using a system alignment check coupon, as discussed in the standard and was found to be less than 3\%; as a consequence, the single transducer was used with confidence. 
Table 3: TMI strain gauge data-sheet (TMIL, 2018)

GFLAB-3-350-70-1LJCT-F

\begin{tabular}{lll}
\hline Gauge length & 3 & $\mathrm{~mm}$ \\
Gauge width & 2.9 & $\mathrm{~mm}$ \\
Backing length & 9.5 & $\mathrm{~mm}$ \\
Backing width & 5 & $\mathrm{~mm}$ \\
Overall thickness & 0.5 & $\mathrm{~mm}$ \\
Metallic foil thickness & 0.05 & $\mathrm{~mm}$ \\
Resistance & 350 & $\Omega$ \\
Objective material & ABS & - \\
\hline
\end{tabular}

Table 4: Digital image correlation main parameters

\begin{tabular}{lcl}
\hline Subset spacing & 30 & pixel \\
Subset radius & 43 & pixel \\
Strain radius & 15 & pixel \\
Lens correction coef. & 0 & - \\
\hline
\end{tabular}

A low elastic strain gauge was used, manufactured by Tokyo Measuring Instruments Lab. A single element strain gauge was considered, from the GF series. The transducers pertaining to this series can be customized choosing in a certain range some geometrical and electrical characteristics, together with the backing material. As discussed in the introduction, small gauge and backing, length and width, were considered. Polyimide (PI) was chosen as the backing material, due to its thermal affinity with PLA and ABS. High resistance transducers were considered in order to limit heating effects. The supplier provided leadwire-integrated sensors, in order to avoid on-site welding. The key specifications (TMIL, 2018) of the strain gauge used are reported in Table 3 for convenience. A fast curing ethyl cyanoacrylate adhesive manufactured by Henkel was used to bond each strain gauge to its specimen. A grid was used to symmetrically place the transducer. A quarter Wheatstone bridge was used to connect each strain gauge to the acquisition unit. For each test, the strains were sampled over time with a frequency of $5 \mathrm{~Hz}$.

\section{DIC Set-Up}

Both Run(A) and Run(B) specimens were monitored during the deformation with DIC. Each specimen had to be prepared before the test took place to allow points tracking. Given that black PLA was printed, a white spry was used to produce a random distribution of grays on specimen's front surface to increment the correlation, as shown in Fig. 6. For the specimens with bonded SG, the gray distribution was produced after the bonding to correlate the entire surface, as visible in Fig. 7. Each test was recorded with a NIKON D3500 DSLR camera, with Nikkor 18-55 mm f/3.5-5.6 G DX 24.2 Megapixel optics at a frame rate of $30 \mathrm{~Hz}$.

\section{Displacement Correlation}

After each test was performed 5 frames per second were extracted from the video so that to have the same acquisition frequency imposed on the testing machine. This also lowered the computational cost and time and reduced the noise oscillation between the displacements measured on two adjacent frames. The frames were transferred to Ncorr, a 2D digital image correlation software running on MATLAB. Table 4 summarizes the DIC parameters that were used in the correlation. The first frame preceding the test was set as the reference image, against which the displacements would have been calculated. A manual draw of the Region Of Interest (ROI) was done, in order to identify the region of the frames to be correlated. A displacements field (longitudinal/transverse) was then obtained per each frame; the displacements are evaluated on an equallyspaced grid points called subsets. In the same points, the software allows a direct calculation of a strains field after the derivation parameters are set. The user is prompted to set a strain radius, which represents the surface dimension over which the displacements gradients are to be evaluated. A small radius is usually sensitive to image noise, while a high radius leads to smooth results. A key difference between DIC and SGs arises here; a SG returns a single signal, a single strain over time, which represents the average value over the surface it is applied to. DIC returns an high number of "strains", instead, as a single strain is calculated in each subset. In order to be comparable, it was necessary to find a way to deal with a single strain also for DIC. In a properly executed tensile test, one would expect a uniform distribution of strains, at least in the linear elastic section of the test. This may not be true once approaching the maximum load, in the plastic deformation region and close to the (potential) necking of the specimen.

For each test, the correlation dealt with a rectangular ROI, characterized by $M \times N$ subsets. $M$ identifies the subset number in the longitudinal direction and $N$ that in the transverse direction. $M$ and $N$ are not the same values among the tested specimens as the ROI was manually drawn. Figure 8 shows an example of outputs of the DIC made for F1 specimen: An ideal linear distribution of the longitudinal displacements Vs. The longitudinal coordinate was found (Pearson coefficient higher than 0.99) under the same transverse coordinate. $N$ linear regressions of the longitudinal displacements Vs. The longitudinal coordinate were performed. In this way, the longitudinal displacements were described by a linear equation, whose first derivative represented the longitudinal strain per "column". A total number of $N$ longitudinal strains were obtained; their mean value was identified as the required strain, representative of the overall behavior of the specimen. This operation was performed per each frame; this led to a single strain Vs. Time relation, as that given by the SG. 


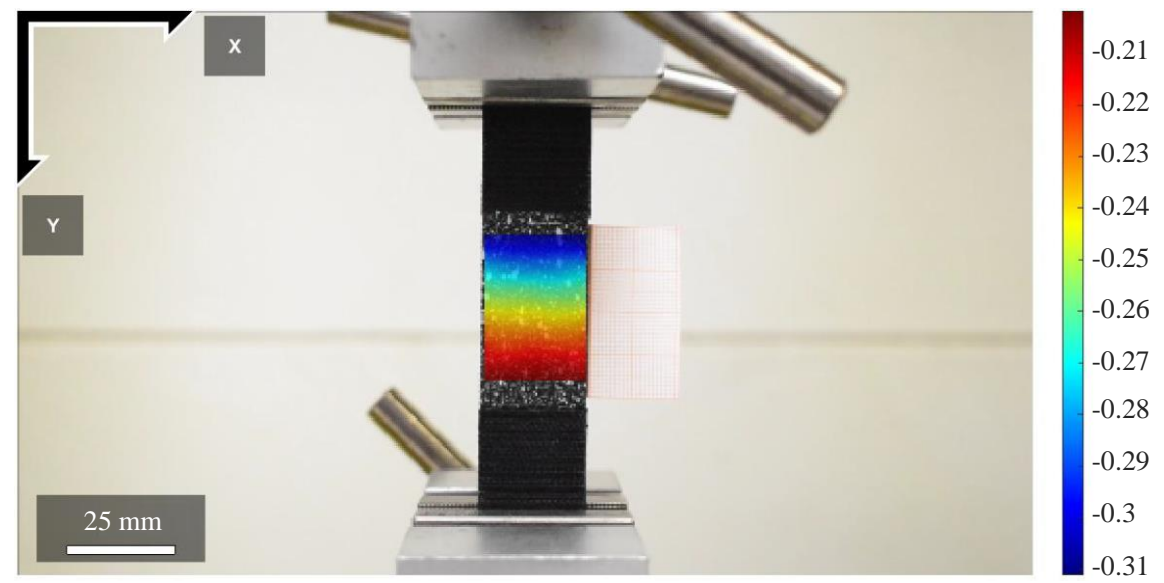

Fig. 8: Longitudinal displacements map for F1 specimen, obtained via DIC

\section{Distortion Check}

Digital image correlation systems are known to be affected by lens distortion, whose impact has been studied by several authors (Lava et al., 2013). Even though it is known that the optic used has no distortion by $35 \mathrm{~mm}$ zoom (Bacovsky, 2020), a preliminary validation was made.

\section{Data Acquisition and Post Processing}

Once the experimental campaign was carried out, Run(A) and Run(B) specimens counted on different sets of data. Run(A) specimens were monitored with DIC; consequently, the following data was collected per item:

- $\quad$ A set of load Vs. Displacement (and time), sampled by the testing machine

- A set of strain Vs. Time, sampled by DIC system

Run (B) specimens were monitored with SG and DIC; the following data was collected per item:

- A set of load Vs. Displacement (and time), sampled by the testing machine

- A set of strain Vs. Time, sampled by DIC system

- A set of strain Vs. Time, sampled by the SG

While the load/displacement sampling started as the test begun, the strains measuring started as the acquisition system was operated, whether DIC or SG. In order to express the Stress Vs. Strain relation for mechanical properties evaluation, the strains were synced over time with the stresses, offsetting the initial sampled values with almost nil amplitude. The Stress Vs. Strain curves for Run(A) specimens are reported in Figs. 9 and 10; the stress is reported by the testing machine, the strains are measured via DIC. The Stress Vs. Strain curves for Run(B) specimens are reported in Figs. 11-13; two sub-figures are presented per each specimen. In both the stress is reported by the testing machine; in the top one, the strain is that measured via DIC, in the bottom one the strain is that sampled by SG. The graph for I4 specimen is missing; in addition to what previously discussed about its slipping during the test, Fig. 14 shows that the strain gauge detached during the test. At the first sight, it seems that all the specimens behaved the same way, despite the run and the way the strain was measured. All the specimens showed the classical linear elastic behavior; shortly before the maximum sustained stress, a more or less pronounced non-linear region is present. As previously discussed, a great scatter was found with respect to the tensile strength. Each tensile stress Vs. Longitudinal strain curve was processed with an appropriate MATLAB script, in order to determine the apparent modulus of elasticity. Excluding the first stress-strain pairs at low loads (below $10 \mathrm{~N}$ ), the script made a linear regression over several ranges of gradually increasing dimension, in order to detect the load at which the linear relation stopped being true. That load was identified as the elastic limit for the specimen and the slope of the linear regression as the apparent modulus of elasticity. Table 5 shows the elastic moduli so determined for Run(A) and Run(B) specimens, obtained using DIC a strain measuring device. The two sets of data are consistent, as obtained on two different Runs, monitored with the same technique. Such a comparison aims to:

- Define the actual tensile modulus of 3D printed PLA, from Run(A) specimens

- Investigate the global reinforcing effect due to the SG bonded to Run(B) specimens

\section{Discussion and Results}

From Run(A) specimens, it was determined that the actual tensile modulus of 3D printed PLA can be evaluated in $3358 \mathrm{MPa}$; there is no discernible difference with Run(B) specimens, for which the mean value $\mu$ stabilized at 3353 $\mathrm{MPa}$. It is worth noting that a similar standard deviation $\sigma$ 
characterized both the distributions, despite the small dimensions of the sample. Table 6 shows the elastic moduli so determined for Run(B) specimens only; while the first column is obtained using the DIC system as a strain measuring device, the second column relies on strain gauges. Also, those two sets are consistent, as obtained on the same Run, but with different monitoring techniques. Such a comparison aims to investigate the local reinforcing effect, making a comparison between the elastic moduli of the same specimens. It appears that, when SGs are used as monitoring devices, higher values of elastic moduli are found. Considering the mean values of the elastic modulus for this run, the local stiffening effect was quantified in $+1.8 \%$.
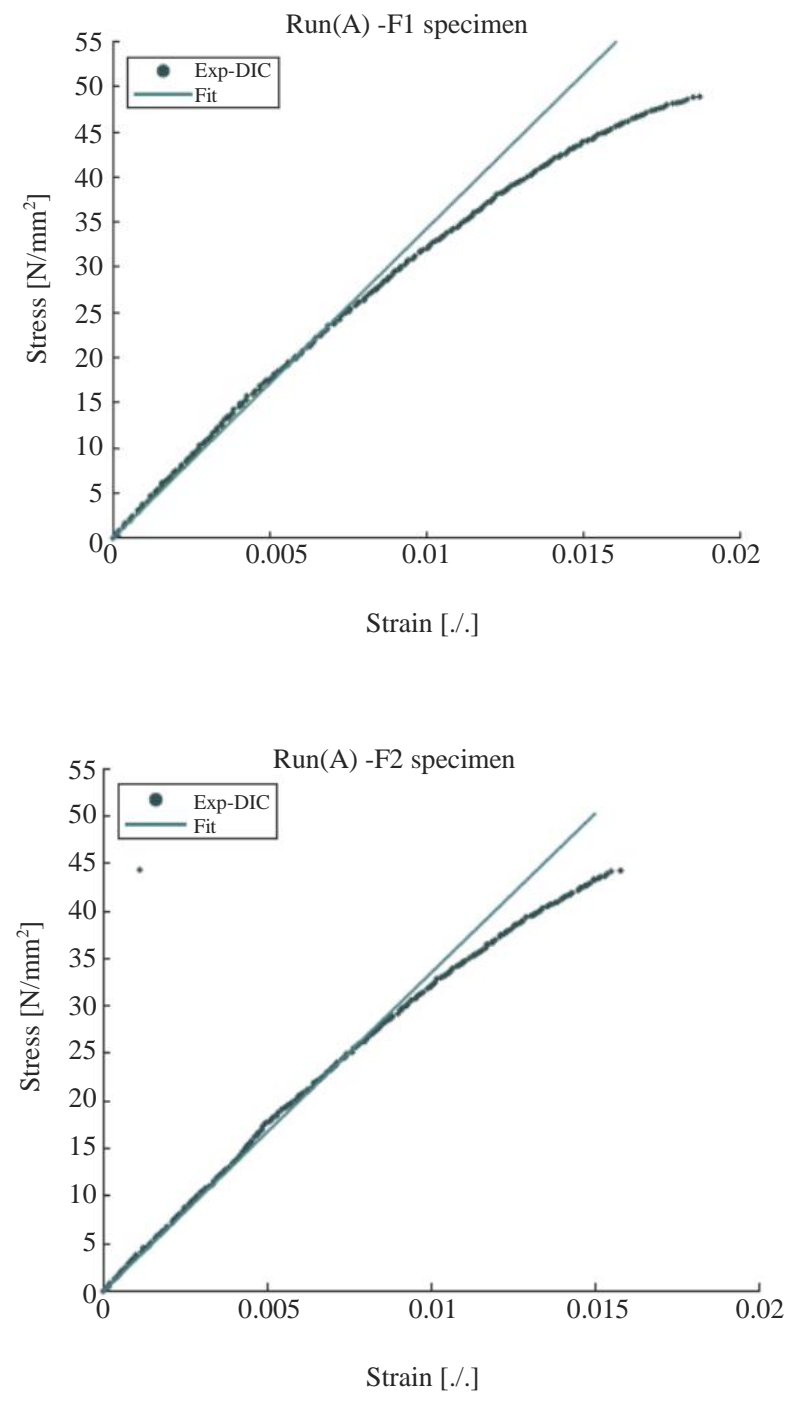

Fig. 9: Run(A): Free (F) specimens; tensile stress Vs. longitudinal strain curves; (a) Run(A) -F1; (b) Run(A)-F2
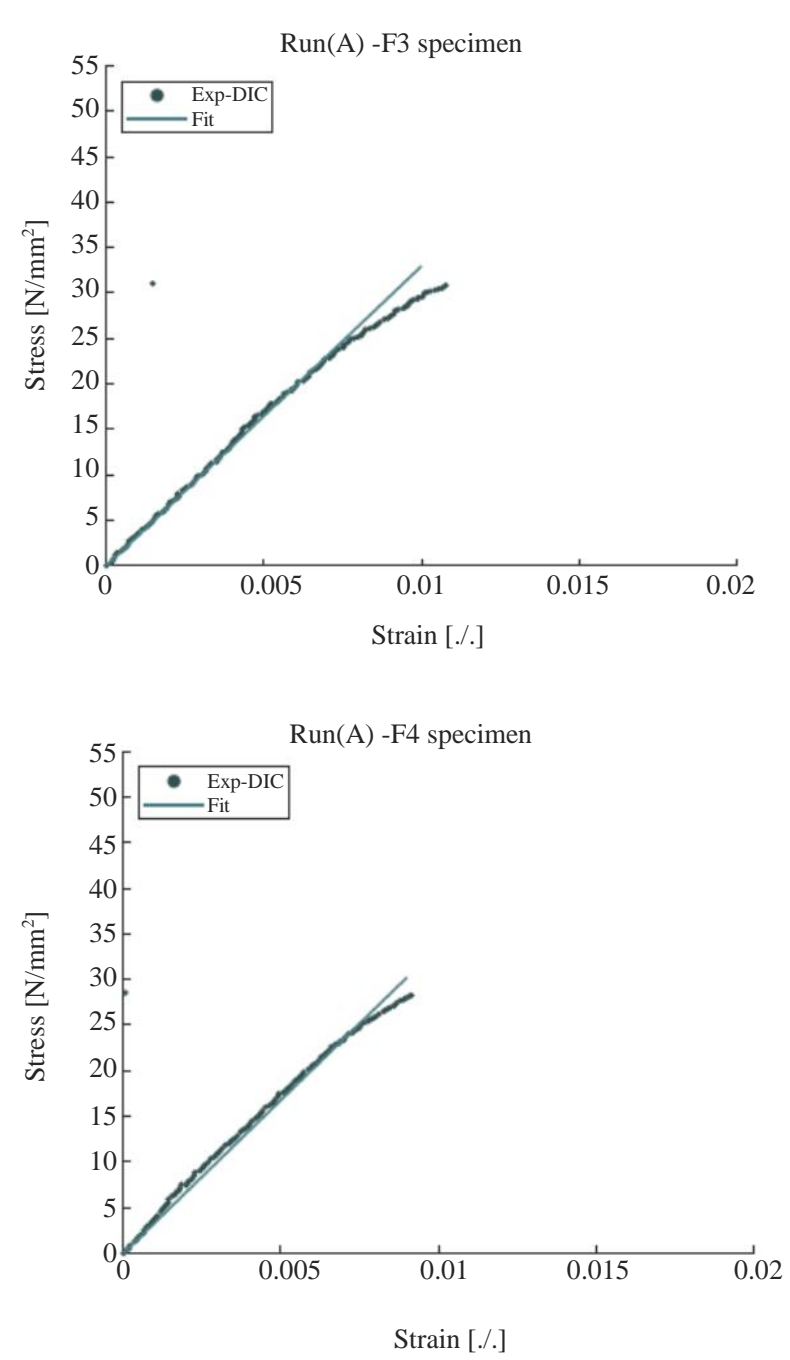

Fig. 10: Run(A): Free (F) specimens; tensile stress Vs. longitudinal strain curves; (a) Run(A) -F1; (b) Run(A)-F2

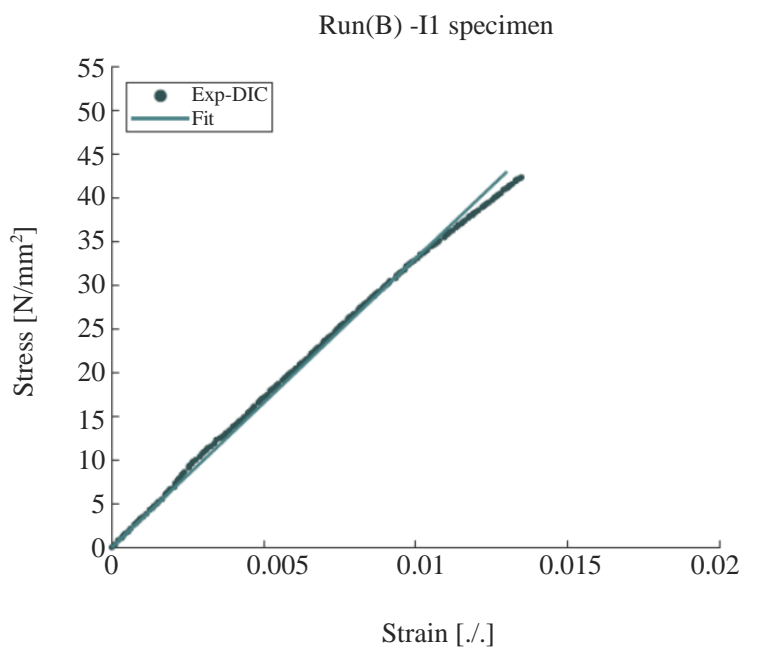




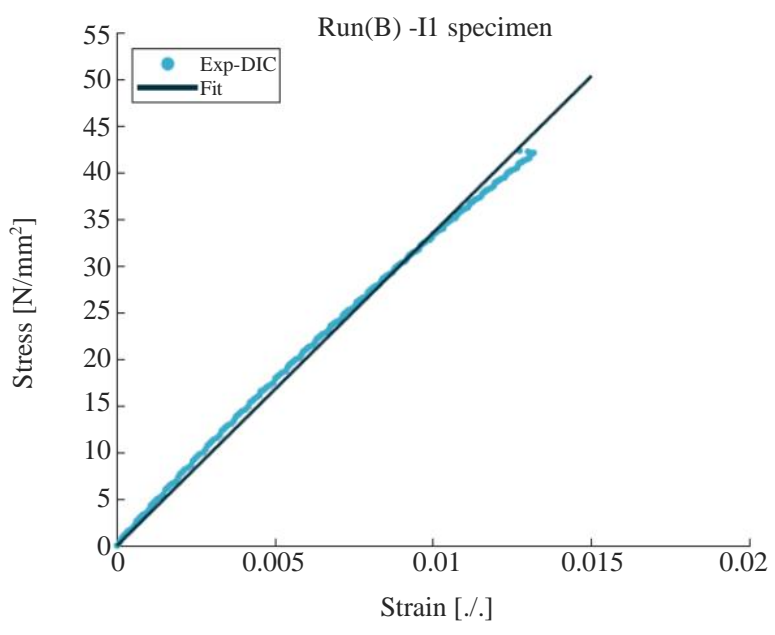

Fig. 11: Run(B): Instrumented I1 specimen; tensile stress Vs. longitudinal strain curves; (a) Run(B) -I1/DIC; (b) Run(B) -I1/SG
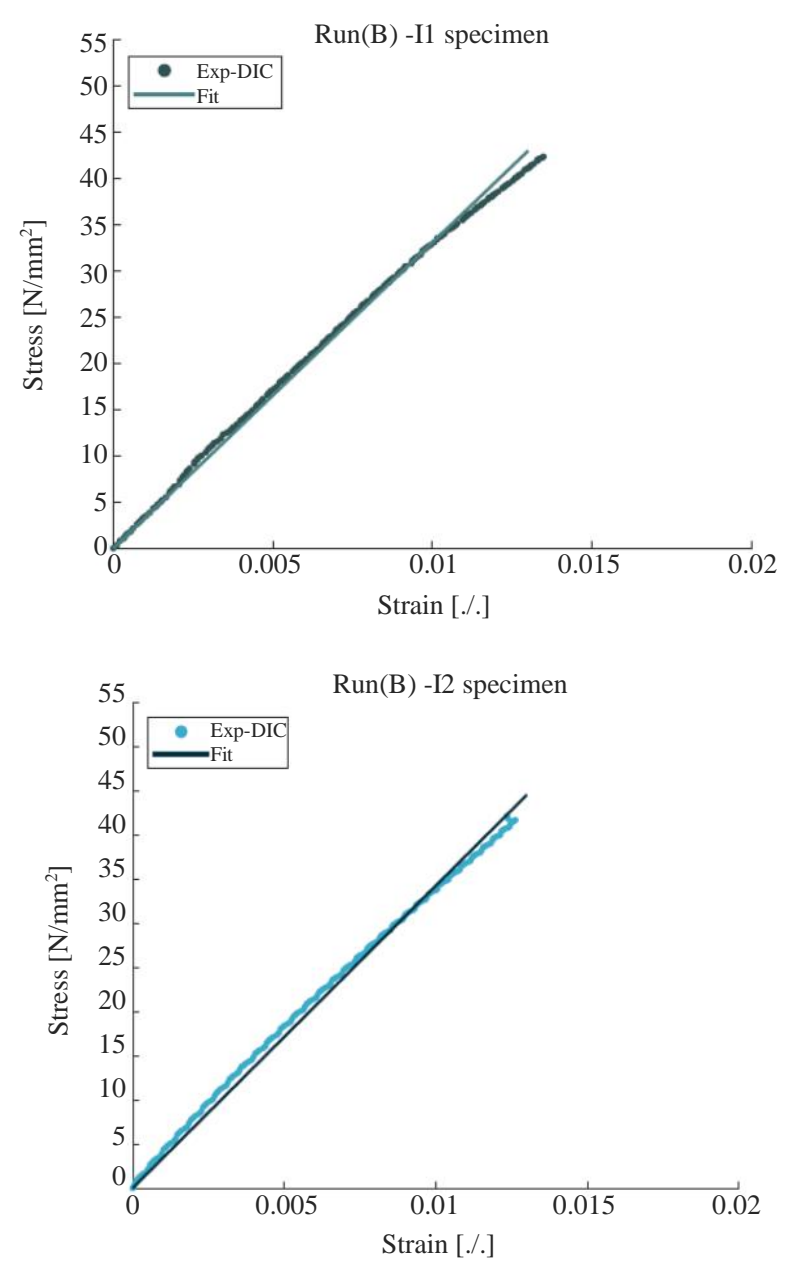

Fig. 12: Run(B): Instrumented I2 specimen; tensile stress Vs. longitudinal strain curves(a) Run(B) -I1/DIC; (b) Run(B) -I2/SG
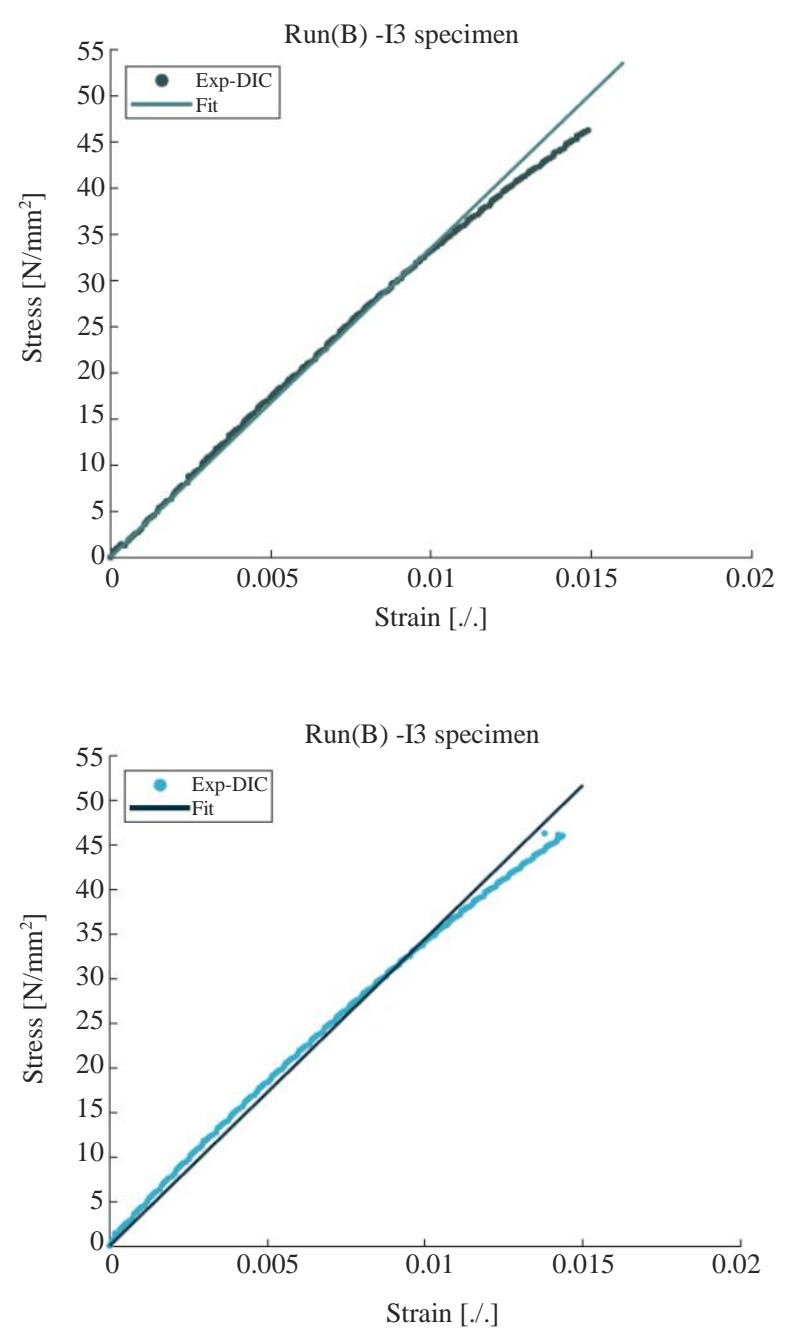

Fig. 13: Run(B): Instrumented I3 specimen; tensile stress Vs. longitudinal strain curves; (a) Run(B) -I3/DIC; (b) Run(B) -I3/SG

Table 5: Elastic moduli for Run (A) and Run(B) specimens, obtained with DIC as strain measuring device

\begin{tabular}{llll}
\hline Run(A) & via DIC & Run(B) & via DIC \\
\hline F1 & $3427 \mathrm{MPa}$ & I1 & $3308 \mathrm{MPa}$ \\
F2 & $3354 \mathrm{MPa}$ & I2 & $3395 \mathrm{MPa}$ \\
F3 & $3294 \mathrm{MPa}$ & I3 & $3355 \mathrm{MPa}$ \\
F4 & $3358 \mathrm{MPa}$ & & \\
\hline Run(A) & $\mu=3358 \mathrm{MPa}$ & Run(B) & $\mu=3353 \mathrm{MPa}$ \\
& $\sigma=55 \mathrm{MPa}$ & & $\sigma=44 \mathrm{MPa}$ \\
\hline
\end{tabular}

Table 6: Elastic moduli for Run(B) specimens, respectively obtained with DIC (left) and SG (right) as strain measuring devices

\begin{tabular}{llll}
\multicolumn{4}{c}{ with } \\
\hline Run(B) & via DIC & Run(B) & via SG \\
\hline S01(RI) & $3308 \mathrm{MPa}$ & S01(RII) & $3362 \mathrm{MPa}$ \\
S02(RI) & $3395 \mathrm{MPa}$ & S02(RII) & $3429 \mathrm{MPa}$ \\
S03(RI) & $3355 \mathrm{MPa}$ & S03(RII) & $3448 \mathrm{MPa}$ \\
\hline Run(B) & $\mu=3353 \mathrm{MPa}$ & Run(B) & $\mu=3413 \mathrm{MPa}$ \\
& $\sigma=44 \mathrm{MPa}$ & & $\sigma=45 \mathrm{MPa}$ \\
\hline
\end{tabular}




\section{FE Model}

A simple FE model is here proposed to further validate the stiffening effect quantified in Section 1. For consistency with the experimental procedure, a tensile test was simulated. The geometry of the specimen was recreated as a rectangular surface, whose dimensions are those of the specimen introduced in Fig. 1. TRIA3 topology was used to mesh the surface; a total number of 840 elements was considered. The dimensions of the specimen and that of the strain gauge differ for an order of magnitude; a non-uniform mesh was imposed through mesh seeds, denser in correspondence and in proximity to strain gauge's location, more coarse elsewhere. In this way, the region influenced by the SG could be studied in-depth, without overloading the model and the solver. The meshed specimen is shown in Fig. 15. 2D Shell properties were applied to this region; the thickness was imposed equal to that of the specimen (Fig. 1). A Linear Elastic constitutive model for material properties definition was set up, with the mechanical properties experimentally determined from $\operatorname{Run}(\mathrm{A})$ specimens in section 1.1 via DIC.

\section{Mechanical Behavior of the Strain Gauge}

The strain gauge also was recreated as a rectangular surface, representing its mean section. An offset between the strain gauge mean surface and that of the specimen was imposed so that, after its thickness has been defined (Table 3), it would have been in contact with the specimen. The strain gauge was meshed using TRIA3 topology, with the same mesh seeds of the specimen's region underneath it. With the aim of configuring a Linear Elastic constitutive model for this region as well, a digital procedure was implemented in order to estimate the mechanical properties of the transducer in use.

The strain gauge is an heterogeneous component, made by (at least) two constituents: The electrical wires and the backing film. In the actual one, the electrical wires are made of Constantan, a Copper-nickel alloy, while the backing film is made of Polyimide. The mechanical properties of the two materials, summarized in Table 7, are quite different. In addition to this, they occupy different volumes and they are characterized by different shapes. This model relies on the hypothesis that the mechanical behavior of the strain gauge might be described in terms of the volume fraction of their constituents. This means drawing an analogy with composite materials, exploiting the well-known rule of mixture. Under this frame, an homogenization of the modeled strain gauge takes place, whose mechanical properties are a function of:

- The mechanical properties of Constantan (the reinforcement)
- The mechanical properties of Polyimide (the matrix)

- The volume fraction of the two constituents

The volume fraction of Constantan and Polyimide is apriori unknown to the final user, while the volume occupied by the strain gauge itself is provided or (at least) easily measurable. The strain gauge may be outlined as a three layered component. The top and the bottom layers are symmetric and made of PI only. The middle layer is made by a combination of Constantan and PI; its thickness is that of the Constantan foil enclosed in the backing, as reported in Table 3 . The front view of the strain gauge allows to clearly detect the Constantan paths, even if the metallic foil does not occupy the strain gauge for its whole thickness. The cross-section dimension of the Constantan foil was digitally evaluated starting from the magnified frontal view of the stain gauge shown in Fig. 16. This picture was carefully cropped to the external strain gauge edges and imported into the vector graphics software Inkscape in order to trace bitmap. Using the Edge Detection tool, the internal path of the strain gauge was recreated. Such a obtained path is reported in Fig. 17; an image containing only black and white pixels was obtained. A Matlab script was then implemented to evaluate the percentage surface occupied by the black path; it turned out that the volume fraction of the metallic wires in the middle layer is $12.67 \%$. Taking into consideration the thickness of the layers, the overall volume fractions of the two constituents were calculated:

$V_{f}^{\text {const }}=1.266 \%$

$V_{f}^{p i}=98.744 \%$

Taking into consideration the mechanical properties of the two constituents reported in Table 7 and the volume fractions reported in Equations 1 and 2, the mechanical properties of the strain gauge were predicted as:

$$
\begin{aligned}
& E^{s g}=V_{f}^{\text {const }} * E^{\text {const }}+V_{f}^{p i} * E^{p i}=4.46 G P a \\
& V^{s g}=V_{f}^{\text {const }} * V^{\text {const }}+V_{f}^{p i} * v^{p i}=0.34
\end{aligned}
$$

\section{Boundary Conditions and FE Solver}

As discussed in the introduction, a layer of adhesive was interposed between the specimen and the strain gauge; this detail was simplified and the contact was modeled imposing an element uniform contact between deformable bodies, taking advantage of what discussed in (Ajovalasit et al., 2007). A perfect bonding between the two was speculated, setting the option Glued (G) in the contact table of the model. The load case was easy to recreate; as described in 
the introduction one end section of the specimen is constrained by the fixed jaw, the other by the movable one. The part of the specimen enclosed in the fixed jaw was constrained limiting all the displacements (in the three orthogonal directions). For the piece of the specimen constrained in the movable jaw, only the two transverse displacements were constrained (the one in $y$ and that in $z$ directions). A fixed displacement in the longitudinal direction $(x)$ was imposed in order to recreate the loading conditions on board the testing machine. A small value was considered, $1 \mathrm{~mm}$, in order not to fall into geometrical nonlinearities. The Linear Static solution SOL101 was used to solve the problem; such a model allowed to simulate:

- The behavior of the Free (F) specimen, turning off the shell properties of the strain gauge and reading the results on specimens' nodes

- The behavior of the Instrumented (I) specimen, with the strain gauge glued on it, reading the results on specimens' nodes

- The behavior of the strain gauge, glued over the specimen, reading the results on its own nodes

\section{Reinforcement Effect Prediction}

A preliminary evaluation of the capabilities of the FE model in predicting the reinforcement effect induced by the strain gauge is here discussed. Figure
18 shows the pattern of the longitudinal strains observed along the specimen's longitudinal axis in the FE model. The blue-dotted curve describes the strains in the specimen, the red-dotted curve those in the strain gauge. This first result allows a qualitative evaluation of the local stiffening effect; at the same longitudinal coordinates, the strains in the transducer are lower than those in the specimen. No difference was found in terms of strains while reading the results from the modeled Free (F) specimen and from the modeled Instrumented (I) specimen. Analogously to the experimental tests, a displacement in an end section of the specimen was imposed. The testing machine recorded the (required) applied load; the SG/DIC measured the strain. Similarly, the FE solver calculated the (required) constraint forces and the strains. However, the FE solver evaluated the constraint forces in the constrained nodes and the strains in the free nodes. To make the modeled and the experimental results to be comparable, the following operations were performed:

- The overall applied load was calculated adding up the longitudinal constraint forces of the constrained nodes

- The overall longitudinal strain was calculated averaging the longitudinal strains of the free nodes over the specimen

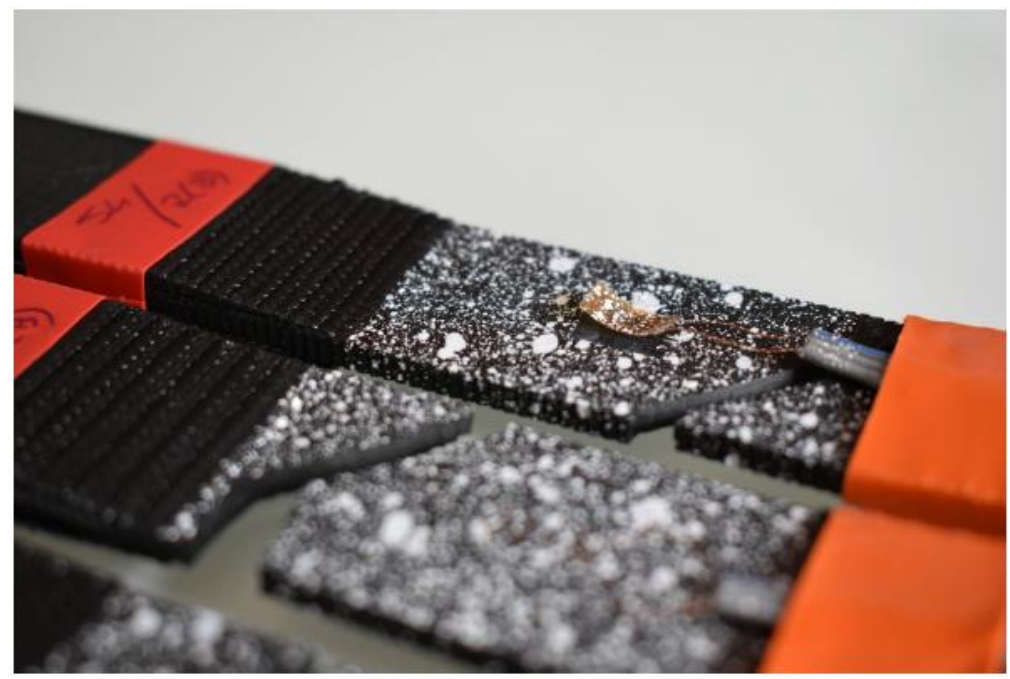

Fig. 14: Run(B) I4 specimen: the strain gauge detached from the specimen during the tensile test

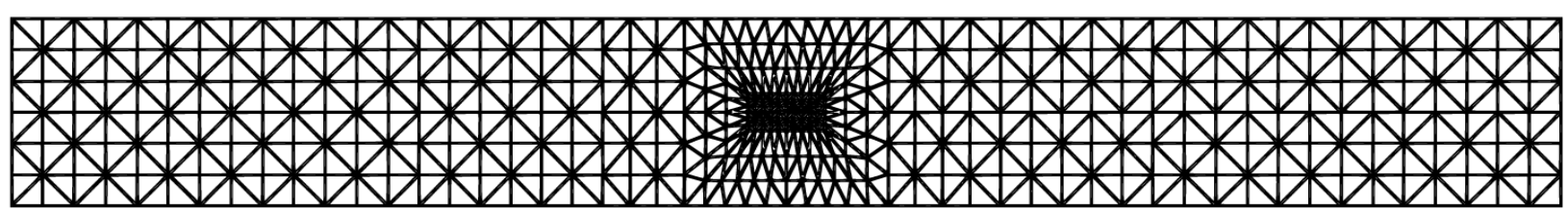

Fig. 15: Meshed specimen 


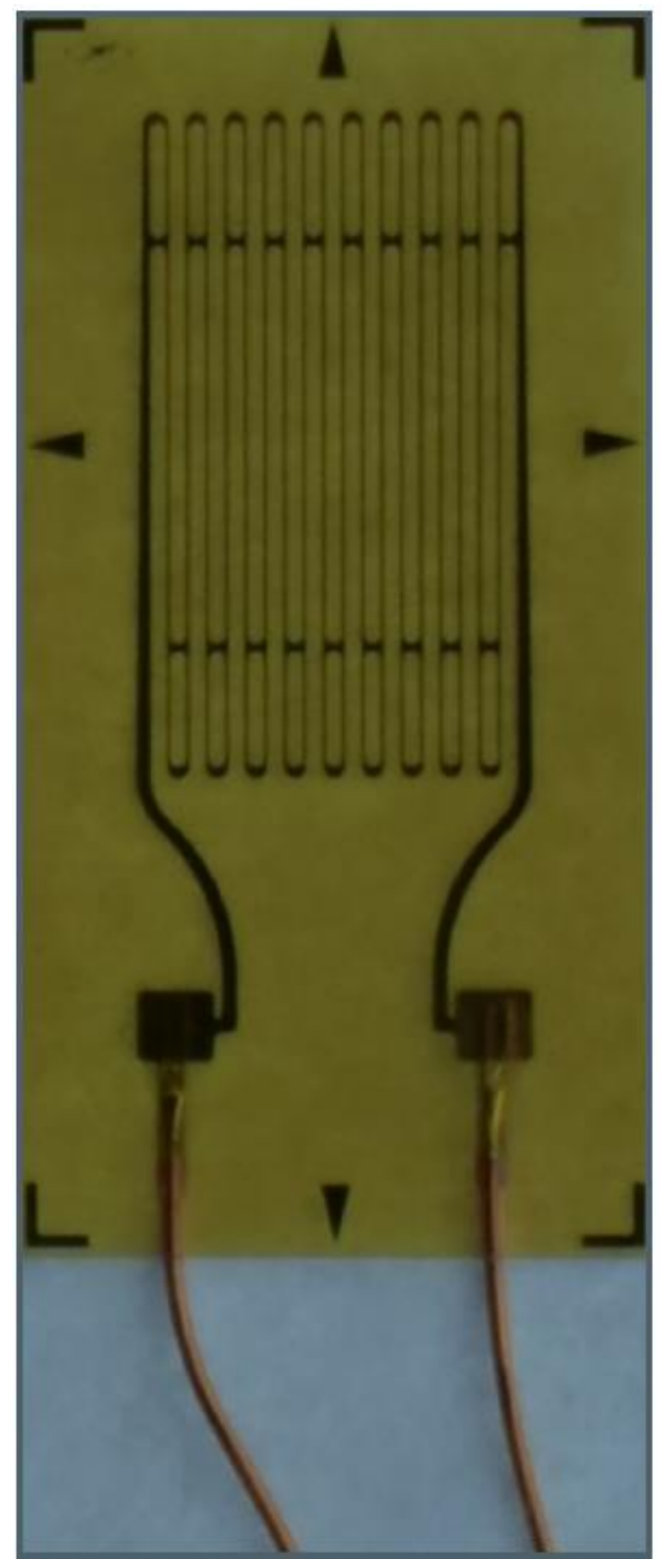

Fig. 16: Front view of GFLAB-3-350-70-1LJCT-F strain gauge

The applied load was then converted into applied stress considering the nominal cross-section dimension of the specimen; the apparent elastic modulus was then obtained taking advantage of Euler's formula. Table 8 summarizes the results. Two apparent moduli of elasticity are given for the modeled Instrumented Specimen, as the strain could be evaluated by means of the specimen's nodes or strain gauge's nodes. As in the experimental tests, no significant difference arose between the Free and the Instrumented specimens, while the strains were evaluated over its surface. At the same time, a $+2.6 \%$ was found in the apparent elastic modulus when the strains measured along the strain gauge were considered.

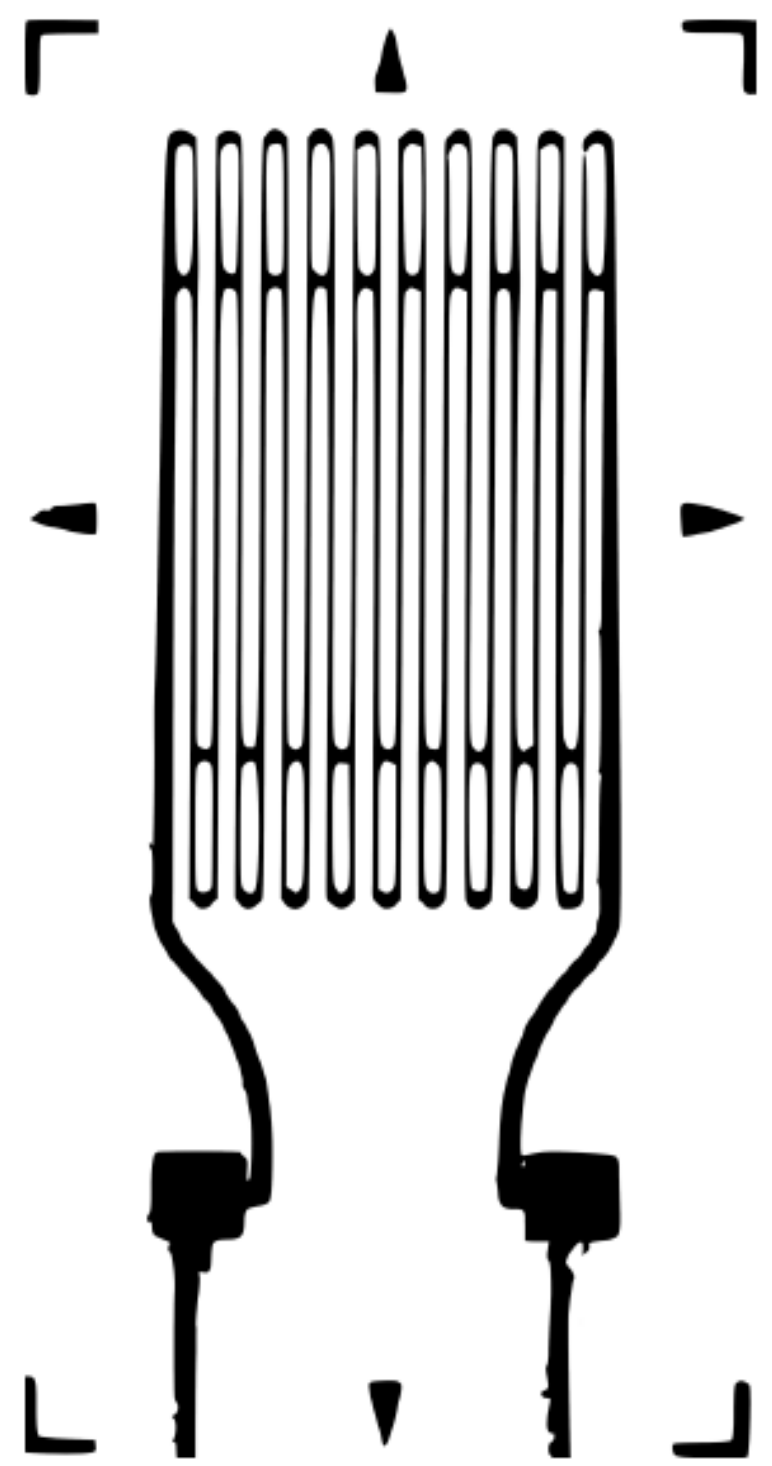

Fig. 17: Internal paths of the strain gauge

Table 7: Mechanical properties of strain gauge constituents (WM, 1972; Dupont, 2020)

\begin{tabular}{lll}
\hline & Constantan & Polyimide \\
\hline Elastic Modulus & $E^{\text {const }}=165 \mathrm{GPa}$ & $E^{p i}=2.5 \mathrm{GPa}$ \\
Poisson ratio & $v^{\text {const }}=0.3$ & $\nu^{p i}=0.34$ \\
Shear Modulus & $G^{\text {const }}=63.5 \mathrm{GPa}$ & $G^{p i}=0.93 \mathrm{GPa}$ \\
\hline
\end{tabular}

Table 8: FE model results. $L$ stands for the applied load, $\sigma$ for the tensile stress

\begin{tabular}{lll}
\hline Free specimen & Instrumented specimen & \\
$L_{F}=2818.1 \mathrm{~N}$ & $L_{I}=2819.4 \mathrm{~N}$ & \\
$\sigma_{F}=37.56 \mathrm{MPa}$ & $\sigma_{I}=37.59 \mathrm{MPa}$ & \\
\hline Over the spcm & Over the spcm & Over the sg \\
$\varepsilon_{F}=0.01117$ & $\varepsilon_{I}^{s m}=0.01117$ & $\varepsilon_{I}^{s g}=0.01089$ \\
$\varepsilon_{F}=3336 \mathrm{MPa}$ & $\varepsilon_{I}^{s m}=3365 \mathrm{MPa}$ & $\varepsilon_{I}^{s g}=3452 \mathrm{MPa}$ \\
\hline
\end{tabular}




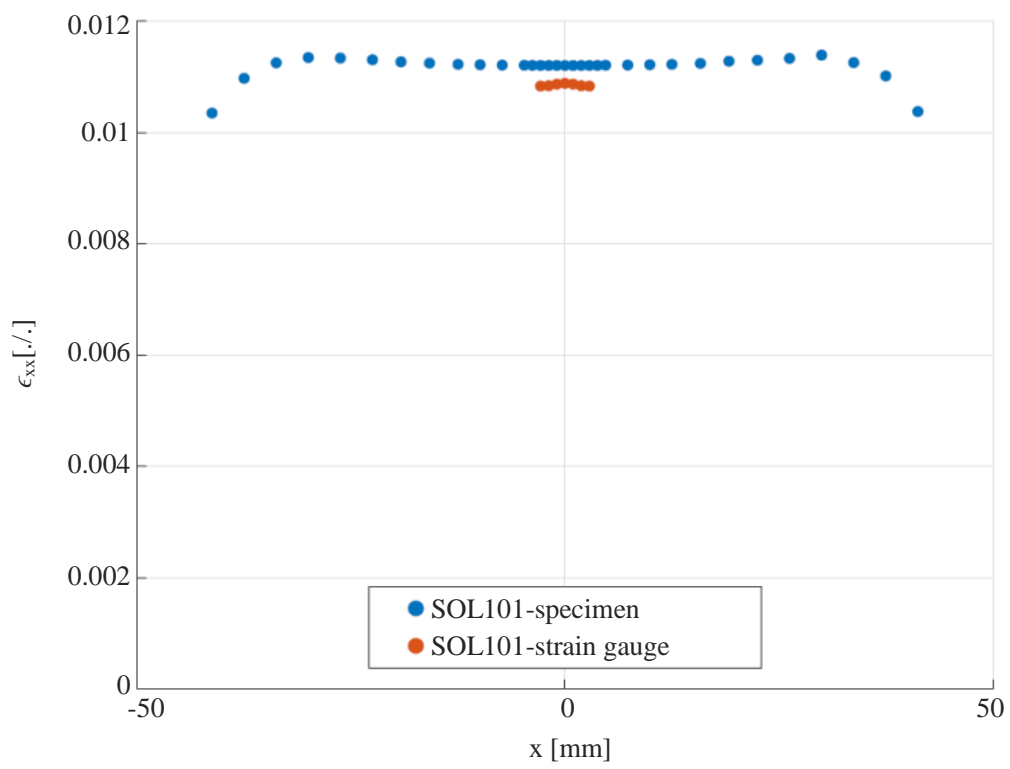

Fig. 18: Longitudinal strains along the loading direction, respectively observed in specimen's nodes and in strain gauge's nodes

Table 9: Local reinforcing effect: Comparison between the experimental results and the prediction offered by the 2D FE and by two analytical models available in the literature

\begin{tabular}{llll}
\hline \multicolumn{2}{l}{ Local Reinforcing Effect } & & \\
Exp. & 2D FE & Beatty & Ajovalasit \\
\hline$+1.81 \%$ & $+2.60 \%$ & $+2.00 \%$ & $+1.99 \%$ \\
\hline
\end{tabular}

\section{Discussion}

The results of the FE model and those obtained experimentally can be directly compared with the empiric formula proposed in (Beatty and Chewning, 1979). For consistency, to assume perfect bonding between the transducer and the specimen the thickness of the adhesive layer can be set to 0 and the shear moduli to $\infty$, leading to parameter $\beta$ equals 0 . Taking into account the geometrical characteristics of the strain gauge reported in Table 3 , the mechanical properties of PLA reported in Table 5 and the predicted mechanical properties of the strain gauge, Equation can be used to calculate the parameter $\alpha$ :

$$
\alpha=0.0278
$$

As discussed in (Beatty and Chewning, 1979), when $L_{s g} / L_{g}>2, r$ equals 0.75 . Given the geometrical characteristics of the strain gauge in use, this relation holds, so that Equation 1 can be used to evaluate the stiffening effect, which equals $+2.00 \%$.

A direct comparison can be also performed with respect to the analytical results that can be derived from (Ajovalasit and Zuccarello, 2005). As discussed in the introduction, the authors disregarded the effects of the adhesive; consequently, this formulation keeps consistent with the assumption in the 2D FE model. The value assumed by the function $\varphi$ in Equation 4 is 2.85 (Ajovalasit and Zuccarello, 2005), considering that $L_{g} / t_{s g}$ $=60$ and $L_{s g} / t_{s g}=190$. Consequently, Equation 4 can be used to estimate the stiffening effect, which equals $+1.99 \%$. A direct comparison is difficult to be performed with (Zike and Mikkelsen, 2014) as FE models are used to predict the phenomenon and a specific strain gauge is considered; furthermore, the results are presented in graphs. Table 9 compares the amplitude of the local reinforcing effect experimentally measured and the predictions so far presented. The two analytical models give practically the same result, which is a very good estimation of the experimental one. The FE model gives a consistent result, even if a bit higher.

\section{Conclusion}

This study analyzed the local and the global reinforcement effects due to strain gauge installed on low modulus materials. 3D printed PLA specimens were used materials with similar mechanical behavior.

The actual modulus of elasticity was measured on a sample of tensile specimens, whose strains were measured using the digital image correlation. This quantification was necessary as the mechanical properties of 3D printed polymers usually differ from those of the raw material, being affected by the processing and by its parameters. A linear elastic modulus of $3358 \mathrm{MPa}$ was found. An identical sample of specimens with a bonded strain gauge was then tested; in this case, strains were measured using both the strain gauge and the digital image correlation. This second sample showed a linear elastic modulus of $3353 \mathrm{MPa}$, 
(standard deviation of $44 \mathrm{MPa}$ ) when the DIC was used as a strain measuring device. However, the linear elastic modulus raised to $3413 \mathrm{MPa}$ (standard deviation of 45 $\mathrm{MPa}$ ) when it was evaluated using the SG as a strain measuring device. The experimental tests showed that no appreciable global reinforcing effect took place, as the elastic moduli evaluated via DIC of the two samples were practically the same, with a similar standard deviation. However, a local reinforcing effect was detected, as the apparent modulus of elasticity was found to be $+1.8 \%$ higher than the actual one when evaluated through the strains measured via SG.

A 2D FE model was proposed to describe the phenomenon and give a numerical quantification of both the local and the global reinforcing effects. The authors described the mechanical behavior of the strain gauge drawing an analogy with reinforced plastics. A digital procedure was implemented to evaluate the volume fractions of the metallic and of the polymeric constituents of the strain gauge; through homogenization, the mechanical properties of the transducer were predicted. The numerical model confirmed the experimental results as a $2.6 \%$ local reinforcing effect was predicted and no global reinforcing effect was measurable. The numerical model however seems to overestimate the phenomenon. This can be partially justified taking into account the tendency of FE to stiffen the models, due to the discretization. Indeed, the modeled free specimen is $+0.3 \%$ stiffer than the actual one.

This study can be considered as a further reminder of the phenomenon: Even strain gauge designed for lowmodulus materials can produce small local reinforcing effects affecting the outcomes. Both the experimental and the numerical results were found to be in-line with the methodologies already available in the literature. This validated the proposed digital procedure for strain gauge elastic modulus estimation and the simple FE model, which can be considered as a quick method of evaluation and correction. A follow-up of the present work will extend this analysis to configurations in which the difference between the elastic moduli is greater

\section{Funding Information}

This research received no specific grant from any funding agency in the public, commercial, or not-forprofit sectors.

\section{Author's Contributions}

Roberto Torre: Conception and design, acquisition of data, analysis and interpretation of data, contribute in drafting the article or reviewing it critically for significant intellectual content, give final approval of the version to be submitted and any revised version.
Salvatore Brischetto: Conception and design, analysis and interpretation of data, contribute in drafting the article or reviewing it critically for significant intellectual content, give final approval of the version to be submitted and any revised version.

\section{Ethics}

This article is original and contains unpublished material. The manuscript is approved by both the author in the present form. No ethical issues are involved.

\section{References}

Ajovalasit, A., \& Zuccarello, B. (2005). Local reinforcement effect of a strain gauge installation on low modulus materials. The Journal of Strain Analysis for Engineering Design, 40(7), 643-653.

Ajovalasit, A., D'Acquisto, L., Fragapane, S., \& Zuccarello, B. (2007). Stiffness and reinforcement effect of electrical resistance strain gauges. Strain, 43(4), 299-305.

Arena, M., \& Viscardi, M. (2020). Strain State Detection in Composite Structures: Review and New Challenges. Journal of Composites Science, 4(2), 60.

ASTM. (2017). Standard Test Method for Tensile Properties of Polymer Matrix Composite Materials. Annual Book of ASTM Standards, ASTM International.

Bacovsky, V. (2020). Review of Nikon $1855 \mathrm{~mm}$ f/3.55.6G DX optics. Photograpylife. https://photographylife.com/reviews/nikon-18$55 \mathrm{~mm}-\mathrm{dx}-\mathrm{vr}-\mathrm{af}-\mathrm{p} / 2$

Beatty, M. F., \& Chewning, S. W. (1979). Numerical analysis of the reinforcement effect of a strain gage applied to a soft material. International Journal of Engineering Science, 17(7), 907-915.

Borri-Brunetto, M., Alessio, M., Barbero, M., Barpi, F., Biagi, V. D., \& Pallara, O. (2016). Stiffening effect of bolt-on transducers on strain measurements. Latin American Journal of Solids and Structures, 13(3), 536-553.

Brischetto, S., \& Torre, R. (2020). Tensile and Compressive Behavior in the Experimental Tests for PLA Specimens Produced via Fused Deposition Modelling Technique. Journal of Composites Science, 4(3), 140.

Brischetto, S., Ferro, C. G., Maggiore, P., \& Torre, R. (2017). Compression tests of ABS specimens for UAV components produced via the FDM technique. Technologies, 5(2), 20.

Campbell, W. R., \& Medbery, A. F. (1947). Performance Tests of Wire Strain Gages V: Error in Indicated Bending Strains in Thin Sheet Metal Due to Thickness and Rigidity of Gage. 
Clark, A. B. (1956). Static and dynamic calibration of a photoelastic model material, CR-39. Washington, DC: Naval Research Laboratory.

Dove, R. C. (1955). Strain Measurement Errors in Materials of Low Modules. In Proceedings of the American Society of Civil Engineers (Vol. 81, No. 5, pp. 1-10). ASCE.

Dupont. (2020). Kapton Polyimide Film General Specifications.

http://www.dupont.com/kapton/general/H-384794.pdf.

Hoffmann, K. (1989). An introduction to measurements using strain gages (No. BOOK). Darmstadt: Hottinger Baldwin Messtechnik.

Jerabek, M., Major, Z., \& Lang, R. W. (2010). Strain determination of polymeric materials using digital image correlation. Polymer Testing, 29(3), 407-416.

Kobayashi, A. (1987). Handbook on experimental mechanics. Englewood Cliffs, NJ, Prentice-Hall, Inc., 1987, 1020.

Lava, P., Van Paepegem, W., Coppieters, S., De Baere, I., Wang, Y., \& Debruyne, D. (2013). Impact of lens distortions on strain measurements obtained with 2D digital image correlation. Optics and Lasers in Engineering, 51(5), 576-584.

Little, E. G., Tocher, D., \& O'Donnell, P. (1990). Strain gauge reinforcement of plastics. Strain, 26(3), 91-98.

Liu, M., Guo, J., Hui, C. Y., \& Zehnder, A. T. (2019). Application of digital image correlation (DIC) to the measurement of strain concentration of a PVA dualcrosslink hydrogel under large deformation. Experimental Mechanics, 59(7), 1021-1032.

Montazerian, H., Rashidi, A., Milani, A. S., \& Hoorfar, M. (2020). Integrated sensors in advanced composites: A critical review. Critical Reviews in Solid State and Materials Sciences, 45(3), 187-238.

Moradi, M., Karami Moghadam, M., Shamsborhan, M., \& Bodaghi, M. (2020). The synergic effects of FDM 3D printing parameters on mechanical behaviors of bronze poly lactic acid composites. Journal of Composites Science, 4(1), 17.

Muniandy, K., Ariff, Z. M., \& Bakar, A. A. (2019). Digital image correlation utilization in measuring displacement and strain during plastic film blowing process: A feasibility study. Measurement, 136, 487-500.
Pereira, G., McGugan, M., \& Mikkelsen, L. P. (2016). Method for independent strain and temperature measurement in polymeric tensile test specimen using embedded FBG sensors. Polymer Testing, 50, 125-134.

Perry, C. C. (1989). Strain-Gage Reinforcement Effects on Low-Modulus Materials. In Manual on Experimental Methods for Mechanical Testing of Composites (pp. 35-38). Springer, Dordrecht.

Shenzhen Eryone Technology. (2019). Eryone Galaxy PLA -Data Sheet. https://cdn3d.niceshops.com/upload/file/Glitter_PLA_Filam ent[2].pdf

Shen, S. Y., Masters, F. J., Upjohn II, H. L., \& Ferraro, C. C. (2013). Mechanical resistance properties of FRP/polyol-isocyanate foam sandwich panels. Composite Structures, 99, 419-432.

Stehlin, P. (1972). Strain distribution in and around strain gauges. Journal of Strain Analysis, 7(3), 228-235.

Swan, J. W. (1973). Resistance strain gauges on thermoplastics. Strain, 9(2), 56-59.

Tian, L., Yu, L., \& Pan, B. (2018). Accuracy enhancement of a video extensometer by real-time error compensation. Optics and Lasers in Engineering, 110, 272-278.

TMIL. (2018). Strain Gauges GFseries - Data Sheet. https://tml.jp/eng/documents/strain_gauge/GFseries. pdf.

Watson, R. B. (2008). 12. Bonded Electrical Resistance Strain Gages. Springer handbook of experimental solid mechanics, 283.

WM. (1972). Copper-Nickel Alloys-Cu Ni44 Mn1. https://copperalliance.eu/uploads/2018/03/cuni44mn 1.pdf.

Xue, G., Fang, X., Hu, X., \& Gong, L. (2018). Measurement accuracy of FBG used as a surfacebonded strain sensor installed by adhesive. Applied optics, 57(11), 2939-2946.

Zike, S., \& Mikkelsen, L. P. (2014). Correction of gauge factor for strain gauges used in polymer composite testing. Experimental Mechanics, 54(3), 393-403. 